\title{
Freie Fibulatransplantation zur knöchernen Rekonstruktion an der oberen Extremität
}

\author{
Teil 1 - Operative Technik
}

Ariane Asmus, Simon Kim, Jens-Ingmar Höpfner, Arnulf Vogel, Karla Vogel,

Frank Eichenauer, Andreas Eisenschenk

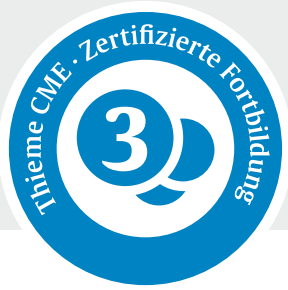

\begin{abstract}
Die Transplantation einer freien, vaskularisierten Fibula zur langstreckigen knöchernen Rekonstruktion kongenitaler, onkologischer oder traumatischer Defekte sowie auch zum Erhalt der Extremität nach Osteomyelitis und frustranem avaskulärem Knochenersatz etablierte sich zunehmend in den letzten 40 Jahren. Die Technik der Fibulahebung und Transplantation ist variabel und soll hier exemplarisch in Form des lateralen Zugangs dargestellt werden.
\end{abstract}

\section{Einleitung}

\section{Epidemiologie und Ätiologie}

Zur Rekonstruktion großer knöcherner Defekte mit dem Ziel des Extremitätenerhalts wurde erstmals 1975 in Australien durch G. I. Taylor eine freie vaskularisierte Fibula (fvF) nach Trauma am kontralateralen Unterschenkel durchgeführt und publiziert [1]. 1981 veröffentlichte Allieu erstmals die Transplantation einer fvF beim Kind zur Rekonstruktion einer kongenitalen Unterarmpseudarthrose [2]. Ebenfalls 1981 beschrieben Hurst u. Mitarb. den ersten klinischen Fall der erfolgreichen Rekonstruktion der Ulna nach chronischem Infekt und weiteten somit die Indikationsstellung bezüglich des Knochenersatzes auch auf die Osteomyelitis aus [3].

Im Jahr 1983 veröffentlichten Ueba u. Fujikawa [4] eine bereits 1973 in Japan durchgeführte freie, vaskularisierte Fibulatransplantation zur Rekonstruktion von einem Drittel der Ulna aufgrund einer onkologisch bedingten Resektion bei einem 11-jährigen Kind als Case Report mit einer 9 Jahre langen Beobachtungszeit [5].

Im Jahr 1988 beschrieb Pho 3 Fälle einer proximalen, freien, vaskularisierten Fibulasegmenttransplantation in die obere Extremität unter Mitnahme der Epiphyse und einer umgebenden Muskelmanschette, welches laut den Autoren die ausreichende Blutversorgung der Epiphyse gewährleisten sollte. Die Ergebnisse zeigten ein verlangsamtes Wachstum, wiesen aber auf ein Überleben der Epiphyse hin [6]. Innocenti veröffentlichte im Jahr 2005 seine Erfahrungen mit der Technik der proximalen, vaskularisierten Fibulasegmenttransplantation bei Kindern seit 1992 und favorisierte für den funktionellen Erhalt der Epiphyse eine ausreichende Blutversorgung unter Mitnahme der A. tibialis anterior als sehr anspruchsvolle Technik, zeigte damit aber auch ein fortschreitendes Längenwachstum nach Transplantation [7].

Das sehr günstige biologische Verhalten eines vaskularisierten Knochentransplantats und die zusätzlich sehr variable Transplantatlänge führten dazu, die Indikationen sukzessive zu erweitern und in Fallserien nachzuuntersuchen, wobei sich die Methodik sowohl im infektiösen und traumatischen als auch im kongenitalen und onkologischen Anwendungsbereich etablierte.

\section{Biologisches Verhalten}

In die Überlegung vor einer Rekonstruktion gehen die am Empfängergebiet vorhandenen lokalen Weichteilverhältnisse (Wirtslager) und die Funktion der Extremität mit ein. Dabei müssen vorangegangene Operationen, die Motivation und Erwartungen des Patienten sowie die Zeit der Heilung und Rehabilitation berücksichtigt werden [8].

Bayne u. Mitarb. beschrieben, dass die vaskularisierte Knochentransplantation eine schnellere Konsolidierung, eine knöcherne Hypertrophie, weniger Ermüdungsbrüche und geringere Resorptionsraten bewirkt als avaskuläre Knochentransplantationen $[9,11]$.

Eisenschenk fasst für seine Untersuchungen von 19911994 zusammen, dass die freie Fibula als vaskularisiertes Transplantat, also ein vitaler kortikaler Knochen mit endostaler und periostaler Blutzirkulation, auf biologische Belastungen wie ein normaler Knochen reagiert 
$[12,14]$. Dieser heilt dementsprechend nach den Gesetzen der Frakturheilung, reagiert als Transplantat mit einer Dickenzunahme und einer normalen Infektresistenz. Eine Einheilung auch im vorgeschädigten Knochenwirtslager, wie z. B. bei Narben, Kontaminations-, Bestrahlungs- und/oder Chemotherapiefolgen, ist erschwert, aber bei ausreichend guter Vaskularisation in der Regel möglich.

Muramatsu fasste seine Beobachtungen im Jahr 2014 mit vergleichbaren Ergebnissen zusammen und beschrieb eine schneller stattfindende Knochenheilung im Vergleich zum avaskulären Transplantat und eine höhere Toleranz gegenüber Infektionen. Die Knochenheilung, welche im vorbestrahlten Umfeld möglich ist, bewirkt nämlich eine Neovaskularisation im anliegenden nekrotischen Knochen. Weiterhin konnte nachgewiesen werden, dass die zu Beginn ersichtliche Hypertrophie aus den transplantierten Zellen resultiert, während die später einsetzende Hypertrophie aus den Empfängerzellen herrührt. Dabei ist die früh auftretende Hypertrophie eine Folge der lokalen Knochenreaktion als Kompensation zur lokal entstehenden Nekrose (innerhalb der ersten 6 Wochen postoperativ) und die späte Hypertrophie mit systemischen Faktoren als Antwort und Adaptation auf die neue Umgebung zu verstehen [5].

Nachgewiesen wurde im Tiermodell, dass in avaskulären Transplantaten nur 5-10\% der oberflächlichen Osteozyten überleben. Der übrige Knochen stirbt innerhalb 1 Woche ab und wird durch eine diffuse Knochenmarkfibrose ersetzt. Die suffiziente Heilung erfolgt dann aufgrund langsam einwachsender neuer Zellen, der sog. „creeping substitution“. In transplantierten vaskularisierten Knochen fand man hingegen bis zu 52,4\% lebende Zellen, sodass die Notwendigkeit einer schleichenden Durchbauung in deutlich geringerem Maß ausfallen konnte $[15,16]$.

\section{Anatomische Besonderheiten}

\section{Größe und Durchmesser des Transplantats und Auswahl der Entnahmeseite}

Die physiologischen Dimensionen der Fibula mit einem Durchmesser von ca. $1,5 \mathrm{~cm}$ und einer Entnahmelänge von bis zu $25-30 \mathrm{~cm}$ erweisen sich besonders günstig für die direkte Rekonstruktion der Ulna und des Radius. Ferner passt die Fibula quasi ideal in den medullären Raum von Humerus, Femur und Tibia. Der zusätzlich hohe Anteil an kortikalem Knochen gewährleistet eine gute Stabilität gegenüber Rotationsstress [17].

Die Entnahme der Fibula erfolgt in der Regel im mittleren Anteil, wobei ein ca. $8 \mathrm{~cm}$ langes Fibulasegment proximal zum Schutz des N. peronaeus, des proximalen fibulotibialen Gelenks, des Ansatzes des M. biceps fe-

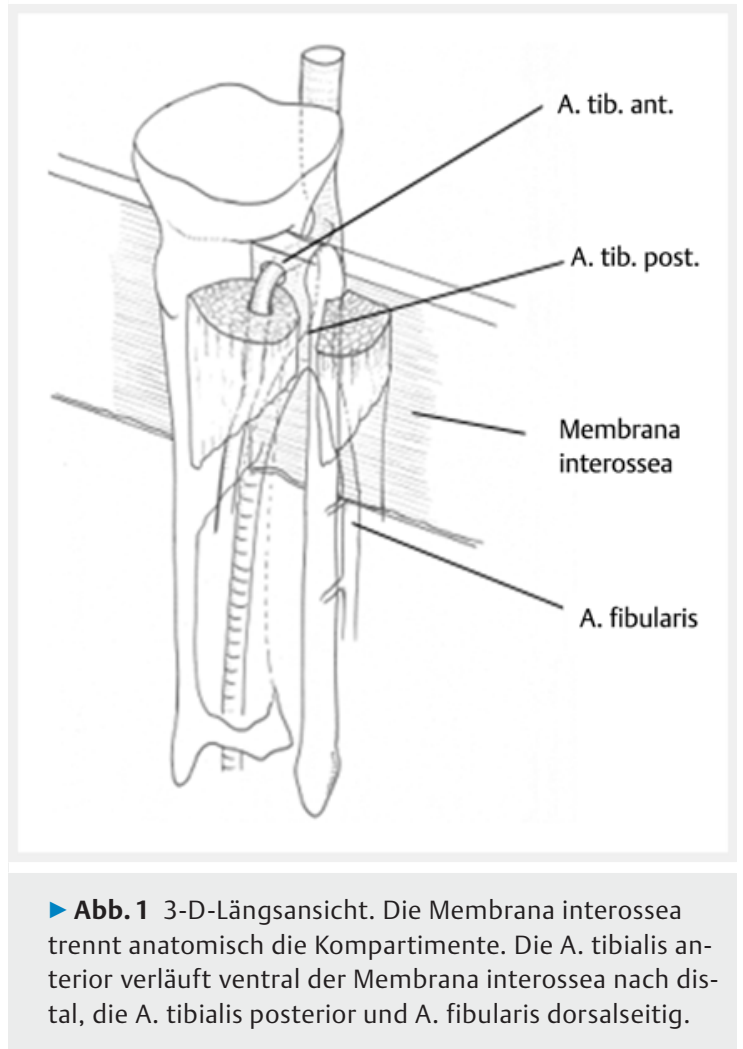

moris und des lateralen Kollateralbands des Kniegelenks sowie ca. $6 \mathrm{~cm}$ distal zur Gewährleistung der Stabilität des Sprunggelenks belassen werden sollten $[9,18]$.

\section{Blutversorgung}

Wie andere lange Röhrenknochen wird die Fibula von einem endostalen und periostalen Gefäßbaum versorgt.

Die A. fibularis entspringt i. d. R. ca. $3 \mathrm{~cm}$ distal des Abgangs der A. tibialis anterior und durchbricht die Soleusmuskulatur. Dabei ist sie adhärent an dem lateralen intermuskulären Septum und verläuft dorsal der Membrana interossea. Sie verläuft dann parallel entlang der Fibula nach distal geschützt zwischen dem M. tibialis posterior und dem M. flexor hallucis longus ( $\mathbf{A b b} \mathbf{1} \mathbf{1}$ ). Die A. fibularis misst zwischen 1,5 und $3 \mathrm{~mm}$ und hat i. d. R. 2 Begleitvenen. Der Pedikel der A. fibularis kann in einer Länge von $6-8 \mathrm{~cm}$ gehoben werden [9].

Die endostale Blutversorgung erreicht die Fibula über das nutritive Gefäß der A. fibularis ca. 6-14cm nach deren Abgang von der Bifurkation. Selten findet sich ein direkter Abgang der A. fibularis aus der A. tibialis posterior. Die A. nutritiva gelangt etwa im mittleren Drittel der Diaphyse in die Fibula und spaltet sich in einen auf- und einen absteigenden Ast, wodurch die Fibula versorgt wird [19]. 
Der Blutfluss verläuft zentrifugal von innen (Spongiosa) nach außen zur Kortikalis.

Die periostale Blutversorgung erreicht die Fibula ebenfalls im mittleren Drittel aus mehreren Ästen der A. fibularis und der A. tibialis anterior [9,20]. Zusätzlich findet sich proximal eine Gefäßarkade von den lateralen inferioren Gefäßen des Kniegelenks und ein Ast der A. tibialis anterior für die Versorgung der proximalen Epiphyse der Fibula [7].

Aufgrund der Blutversorgung sowohl endostal als auch periosteal sind knöcherne Anpassungen durch Osteotomie in Längsrichtung als auch „Closed-Wedge-Osteotomien“ zur Rekonstruktion von komplexen anatomischen Verhältnisse wie zum Beispiel im Bereich der Mund-, Kiefer- und Gesichtschirurgie unter Erhalt der Durchblutung möglich.

\section{Perforansgefäße}

Mehrere fasziokutane und myokutane Perforansgefäße, auch Perforatoren genannt, welche von der A. fibularis ausgehen, ermöglichen es, eine zusätzliche Hautinsel mit einer Größe von maximal $10 \times 20 \mathrm{~cm}$ zu heben [21]. Grundsätzlich können diese Perforatoren auch vorab mit einer Doppler-Untersuchung lokalisiert werden.

Die Perforatoren treten im mittleren Drittel der Fibula durch das dorsale Drittel des M. peronaeus longus, während sie sich im distalen Drittel eher zwischen dem M. soleus und M. peronaeus longus befinden können.

\section{Cave}

Insbesondere beim Durchtritt der Perforatoren durch den M. peronaeus longus ist beim Heben einer zusätzlichen Hautinsel darauf zu achten und die Perforatoren sind zu schonen, wenn der Zugang zur Fibula zwischen dem M. peronaeus longus und M. soleus gewählt wird.

Die übliche Technik wird dabei insofern modifiziert, dass die Perforatoren anterior und posterior dargestellt werden und diese mit einer begleitenden Muskelmanschette des M. flexor hallucis und des M. peronaeus longus im proximalen bis mittleren Drittel sowie im distalen Drittel mit einer Muskelmanschette des M. flexor hallucis und des M. soleus gehoben werden [9]. Der M. soleus kann auch als zusätzliche Muskellappenplastik am gleichen Gefäßbaum entnommen werden, um beispielsweise weitere Defekte zu verschließen und Totraum zu obliterieren.
Entnahme der proximalen Epiphyse

Des Weiteren ist auch die Mitnahme der proximalen Epiphyse bei Kindern für ein weiteres Längenwachstum möglich. Dabei sind 2 technische Besonderheiten zu erwähnen, die Hankiss wie folgt beschrieb $[7,8]$ :

- Die zusätzliche Entnahme der A. tibialis anterior beim Kind, da eine alleinige Versorgung der Epiphyse durch die A. fibularis zum vorzeitigen Epiphysenschluss führen würde. Es ist daher notwendig, bei Mitnahme der Epiphyse eine zusätzliche Anastomose mit der A. tibialis anterior durchzuführen.

- Nach Entnahme des Caput fibulae Refixierung des Lig. collaterale laterale an der Tibia zur Stabilisierung des Kniegelenks.

Eine klinische Nachuntersuchung von Kindern mit Lowund High-Grade-Osteosarkomen des proximalen $\mathrm{Hu}$ merus mit kompletter Resektion des proximalen $\mathrm{Hu}$ merus und Transplantation der proximalen Fibula inklusive der Epiphyse wurde von Shammas u. Mitarb. durchgeführt. Bei allen Patienten fand sich ein Nachweis von Längenwachstum mit einer Wachstumsrate von $0,54 \mathrm{~cm} \pm 0,18 \mathrm{~cm} /$ Jahr [22].

\section{Therapeutisches Vorgehen}

Begonnen wird mit der Beurteilung der Komplexität des zu rekonstruierenden Areals bezüglich Lokalisation, Länge, begleitendem Weichteilschaden und Infektionsgeschehen.

Im Rahmen vorher durchgeführter Débridements ist es entsprechend der Grundlage aller Infektsanierungen notwendig, eine radikale Resektion des avitalen Knochen- und Weichteilgewebes durchzuführen [23]. Dabei besteht die Notwendigkeit mehrerer Abstriche, der Abklärung der mikrobiellen Besiedlung und ggf. Sanierung, insbesondere auch im Hinblick auf Pilzinfektionen, wobei hier eine schnellere und genauere Diagnostik über die Einsendung histologisch zu untersuchender Gewebeproben zu empfehlen ist. Die zwischenzeitliche Defektdeckung kann mittels Vakuumversiegelung oder anderer Verfahren der temporären Weichteildeckung erfolgen. Erst nach abgeschlossener knöcherner Stabilisierung, Infektsanierung und Nekrosektomie sollte eine Abklärung der Gefäßanatomie mit der Frage von mikrovaskulären Anschlussmöglichkeiten für vaskularisierte Transplantate durch eine Angiografie und/oder Doppler-Sonografie erfolgen.

\section{Präoperative Angiografie}

Eine präoperative Angiografie erachten wir als essenziell für das zukünftige Empfängergebiet, da sowohl durch den Unfall als auch bereits vorbestehende Veränderungen (Atherosklerose, Gefäßanomalien, Infektionen) Einschränkungen und Veränderungen der Gefäßanatomie vorliegen können. 
Die Notwendigkeit einer präoperativen Angiografie der geplanten Entnahmeregion ist immer wieder Gegenstand von Diskussionen. Lutz u. Mitarb. untersuchten 1998 in einer prospektiven Studie 120 Patienten mit präoperativer Evaluation mittels gefühltem Pulsstatus und Angiografie. Dabei fanden sich bei 114 Patienten normal palpable Pulse und 6 Patienten, bei denen nur ein Gefäß palpabel war. Von diesen 6 konnten bei 5 Patienten dopplersonografisch die Gefäße dargestellt werden.

In den durchgeführten Angiografien fanden sich in 3\% Abnormalitäten:

- 4-mal eine Hypoplasie einer der 2 Hauptgefäße,

- eine A. fibularis magna,

- ein Fehlen der A. fibularis und Hypoplasie A. tibialis posterior,

- ein Hämangiom der A. fibularis.

Zusammenfassend empfehlen die genannten Autoren die Palpation und Dokumentation der Gefäße des geplanten Spenderbeins, möglichst mehrfach im Rahmen der Vorbesprechung, Aufnahme und präoperativ. Eine Angiografie wird dementsprechend nur bei abnormem Pulsstatus und vorangegangenen Traumata an dem geplanten Entnahmebein empfohlen [24].

Oxford u. Mitarb. empfahlen 2005 als Screening eine MR-Angiografie und bei Nachweis einer 2-Gefäß-Versorgung eine anschließende konventionelle Angiografie [25].

Wir empfehlen im Rahmen der durchzuführenden Angiografie des Empfängergebiets begleitend die angiografische Darstellung des bzw. der möglichen Spenderregion(en), am besten beider Beine, da dadurch eine bestmögliche Aussage auch der Gefährdung des Spenderbeins und versorgungsverzögernde Komplikationen weitgehend abgeschätzt und dementsprechend vermieden werden. Alternativ kann eine Doppler-Sonografie aus entsprechend erfahrener Hand die Angiografie des Spenderbeins ersetzen. Bei bekannten Gefäßvorerkrankungen sowie bei Z.n. Unfällen am Spenderbein halten wir die Angiografie jedoch für unerlässlich.

\section{Wahl der Entnahmeregion}

Stellt sich nach der o.g. Diagnostik die Wahl, welche Spenderregion favorisiert werden sollte, sind die folgenden Punkte zu beachten:

- Bei Ersatz des Femurs empfiehlt sich die Verwendung der ipsilateralen Seite, da grundsätzlich das Risiko oder Möglichkeit der Amputation bei Versagen bestehen kann [19].

- Zum Ersatz der oberen Extremität sollte die Seite entsprechend der Richtung des Gefäßpedikels ausgewählt werden [19].
- Bei der Rekonstruktion am Unterarm aus lagerungstechnischen Gründen Wahl des Spenderbeins kontralateral, um ein Kippen des Beckens zu ermöglichen, ohne Beeinträchtigung der Präparation am kontralateralen Unterarm.

\section{Indikationen/Kontraindikationen}

In engem Kontakt mit dem Patienten sollte nach entsprechenden Wünschen und Erwartungen die Begleiterkrankungen und auch die Möglichkeit einer Amputation diskutiert werden. Dabei ist auf die lange Behandlungsdauer von bis zu 1 Jahr und insbesondere begleitende Nervenschäden (Verlust der Sensibilität der Fußsohle bei Rekonstruktionen an der unteren Extremität) sowie mögliche Vorteile bei einer Prothesenversorgung einzugehen.

\section{Allgemeinzustand des Patienten und Begleit- erkrankungen}

Hierner fasste 2009 zusammen, dass allgemein für die freie, vaskularisierte Knochentransplantation als Kontraindikation gelten [26]:

- ausgeprägte Gefäßveränderungen im Spender- und Empfängergebiet, vor allem keine Entnahme des Transplantats bei $50 \%$ iger Stenose eines der versorgenden US-Gefäße [25],

- systemische Gefäßerkrankungen,

- fehlende Operabilität des Patienten bei schweren Allgemeinerkrankungen und hohem anästhesiologischen Risiko.

\section{Möglichkeit des Gefäßanschlusses}

Neben der Voraussetzung der Beherrschung des lokalen Infektgeschehens steht die Beurteilung der gefäßabhängigen Anschlussmöglichkeiten. Je nach Gefäßangebot sollten die Überlegungen bezüglich einer Endzu-End-, einer End-zu-Seit-Anastomose sowie auch die Möglichkeit eines zusätzlichen Venenloops mit einer Anschlussmöglichkeit weiter proximal evaluiert werden.

Das Anastomosengebiet sollte im Gesunden geplant werden, da aus den vorangegangenen häufigen Débridements erhebliche Vernarbungen und ein Verlust der Elastizität der Gefäße resultieren können [27].

\section{Knöcherne Defektlänge}

In der Literatur werden Mindestdefektlängen von 5-6 $\mathrm{cm}$ definiert. Die meisten Autoren gehen dabei von einer Länge von mindestens $6 \mathrm{~cm}$ aus, insbesondere dann, wenn begleitende Umstände wie Vernarbungen, Infekte, Bestrahlung oder Chemotherapie dazukommen und eine sichere Vaskularisation angestrebt werden muss [14, 28]. 
Die maximale Defektlänge wird i. d.R. mit bis zu $25 \mathrm{~cm}$ angegeben, kann jedoch auch länger (bis zu $30 \mathrm{~cm}$ ) ausfallen $[9,14]$.

Inzwischen werden in der Literatur auch kürzere gefäßgestielte Fibulatransplantate beschrieben und wir konnten ebenfalls im eigenen Patientengut auch kürzere Defektstrecken von nur $4 \mathrm{~cm}$ am Unterarm mit ausreichend guter Vaskularisation und erhaltenem Periostmantel erfolgreich mit einem Fibulatransplantat überbrücken, bei welchen zuvor durch Anlagerung von avaskulären Transplantaten keine knöcherne Konsolidierung erreicht werden konnte [29].

Planung mit zugehörigem Weichteil-/Hautmantel und/oder zusätzlicher Spalthauttransplantation

Merke

Begleitende Weichteildefekte stellen keine Kontraindikation zur Transplantation einer vaskularisierten Fibula dar [30].

In derartigen Fällen ist es erforderlich, die Empfängerregion bezüglich der Knochen-, Weichteil- und Gefäßanatomie für die zusätzliche Rekonstruktion der Weichteile zu kennen. Neben der Möglichkeit einer weiteren freien Lappenplastik besteht die Möglichkeit der Entnahme einer Muskel-/Hautmanschette im Sinne eines freien, vaskularisierten, osteokutanen Fibulatransplantats, wobei die Hautinsel bis zu ca. $20 \mathrm{~cm}$ Länge und $10 \mathrm{~cm}$ Breite messen kann [21].

Dabei ist ein primärer Hautverschluss nach Entnahme am Unterschenkel in Abhängigkeit von den lokalen Verhältnissen meist nur bis zu einer Breite von $5-6 \mathrm{~cm}$ möglich [21]. Größere Entnahmeregionen können aber direkt mit einem Spalthauttransplantat erfolgreich gedeckt werden.

\section{Aufklärung}

Allgemein sollte über eine primär lange Operationsdauer aufgeklärt werden mit ggf., in Abhängigkeit der Begleiterkrankungen, einem Aufenthalt auf der Wachbzw. Intensivstation für 1-2 Tage. Weiterhin zu besprechen sind eine anschließende Bettruhe von mind. 5-7 Tagen und die Antikoagulation mit niedermolekularem Heparin mit gewichtsadaptierter Dosierung während des stationären Aufenthalts.

Weiterhin sollte über eine zusätzlichen Spalthaut- oder Vollhautentnahme von bislang nicht unmittelbar betroffenen Körperregionen mit dem Risiko unschöner Hebedefekte und vermehrte Narbenbildung aufgeklärt werden. Darüber hinaus ist immer über eine zusätzliche Venenentnahme bei intraoperativ unzureichend vorhandenem Gefäßstiel zu informieren.
Für das Spendergebiet ist auf die Möglichkeit einer Schwellung und Nachblutung und das seltene Risiko der Instabilität im oberen Sprunggelenk sowie der Schädigung des N. peronaeus profundus bei Fibulaentnahme hinzuweisen.

Bei Entnahme einer zusätzlichen Muskelmanschette oder des M. soleus besteht zudem das Risiko einer Kraftminderung und Beugeschwäche der Großzehe.

Die früher oft durchgeführte Ruhigstellung der Spenderextremität wird nicht mehr durchgeführt. Eine Mobilisation des Spenderbeins ist postoperativ schmerzadaptiert, aber uneingeschränkt nach Aufheben der Bettruhe möglich.

Für das Empfängergebiet ist insbesondere auf den Verlust des(r) Transplantate(s) und die Rückzugsmöglichkeiten mit Erwähnung weiterer Spenderknochen, wie zum Beispiel die Entnahme am anderen Bein, ggf. die Entnahme eines vaskularisierten Beckenkamm- oder Skapulatransplantats hinzuweisen. Des Weiteren ist über eine verzögerte Frakturheilung und das Risiko einer Ermüdungsfraktur vor allem der unteren Extremität sowie die Option zusätzlicher Implantate inklusive des Fixateur externe aufzuklären.

\section{Operative Verfahren}

Planung in Abhängigkeit von den notwendigen operativen Verfahren wie zusätzlicher Venenentnahme, Hauttransplantationen usw. mit einer Operationszeit von ca. 6-10 Stunden, möglichst mit 2 Operationsteams:

- Ein Team bereitet das Transplantatgebiet mit Débridement und Darstellen der Gefäße vor.

- Ein zweites Team beginnt mit Präparation der Fibula.

Perioperativ erfolgt das gemeinsame Planen der knöchernen Fibulalänge anhand der anatomischen Gegebenheiten und der Länge des Gefäßstiels.

Begleitend erfolgt eine perioperative Antibiotikaprophylaxe entsprechend den hausinternen Richtlinien bzw. dem evtl. bekannten Keimspektrum des Patienten mit einer Wiederholungsgabe nach 6-8 Stunden.

\section{Entnahme der Fibula}

Taylor beschrieb die Entnahme bzw. Hebung über einen dorsalen Zugang als muskuloossäres Transplantat, wobei inzwischen deutlich häufiger der laterale Zugang von Gilbert 1979 genutzt wird ( $>$ Abb. 2) [17, 19]. Zur Anwendung kommt auch die modifizierte Version von Wood mit einem Zugang zwischen dem M. peronaeus longus und $M$. soleus [9]. 


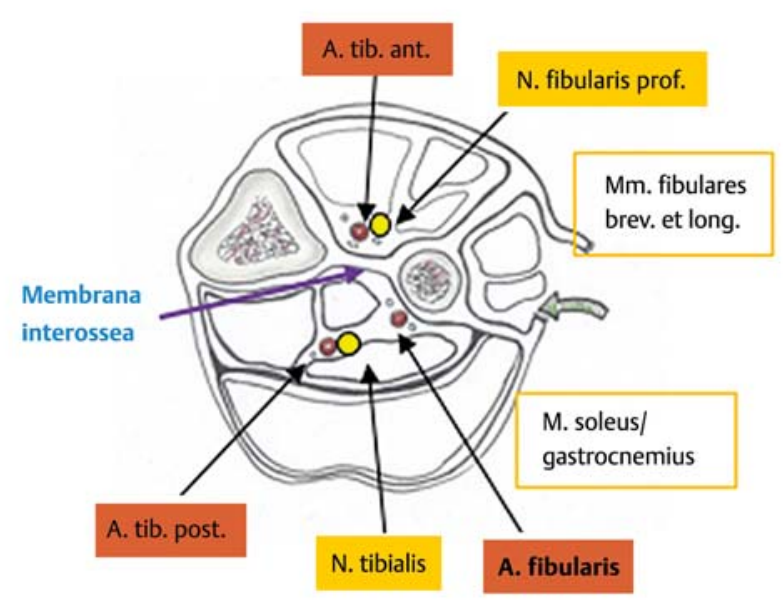

- Abb.2 Querschnitt durch den Unterschenkel im proximalen Drittel mit Lagebeziehungen der versorgenden Gefäße und Nerven in Bezug auf die Kompartimente. Der Zugangsweg ist mit einem Pfeil markiert.
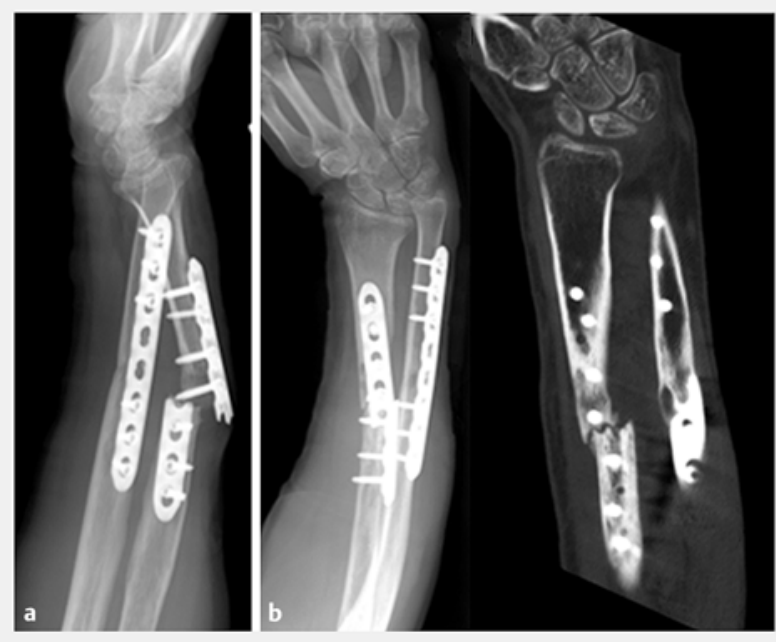

- Abb. 3 Pseudarthrose am Radius am Übergang vom mittleren zum distalen Drittel mit Plattenbruch nach mehrfachen Osteosynthesen mit Spongiosaplastik vom Beckenkamm. a Röntgenaufnahme seitlich. b Röntgenaufnahme a. - p. und Computertomografie.

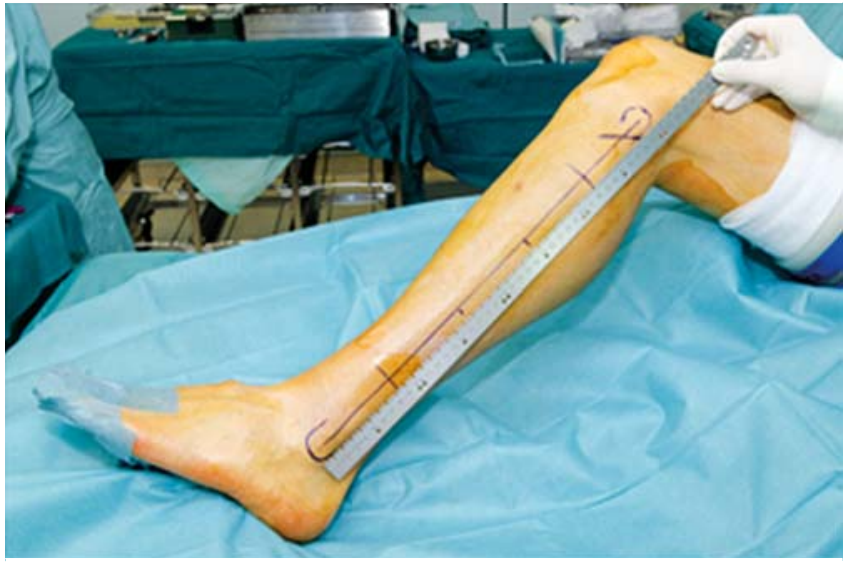

- Abb. 4 Lagerung des Entnahmebeins mit steriler Blutsperre, um eine mögliche Spalthautentnahme vom Oberschenkel zu gewährleisten. Angekipptes Becken. Fußrolle zur Stabilisierung und Lagerung in gebeugter Stellung des Kniegelenks. Abmessen der Fibulalänge und Unterteilung in Fünftel. Markierung des Verlaufs des $\mathrm{N}$. fibularis superficialis et profundus am Fibulakopf. Hebung in diesem Beispiel ohne Blutsperre, falls erforderlich Anlegen mit $300 \mathrm{mmHg}$.

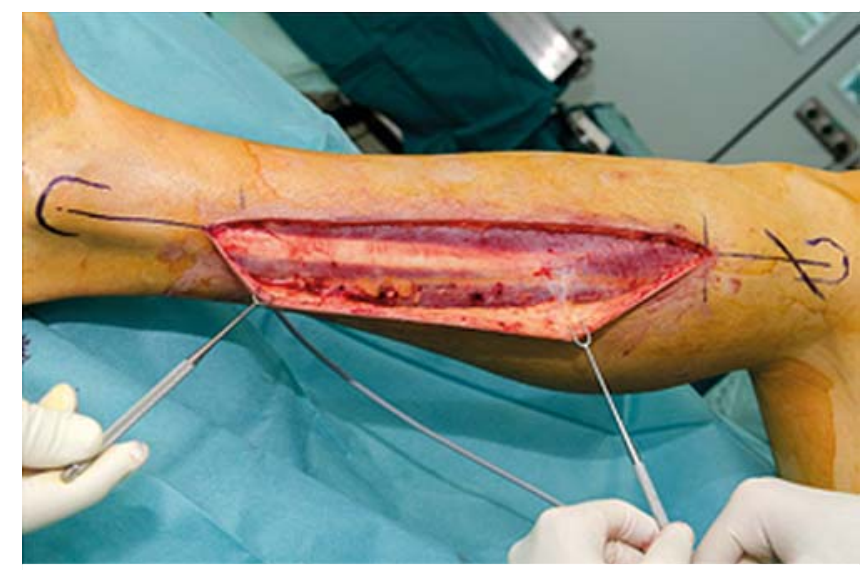

Abb. 5 Inzision der Haut und des Subkutangewebes bis zur oberflächlichen Muskelfaszie. Deutlich sichtbar sind die Mm. fibularis brevis et longus und die (Peroneal-)Sehnen sowie die durch die Faszie erkennbare darunterliegende Abgrenzung zum M. soleus mit einer Fettschicht. Proximal-dorsal spannt sich zwischen Faszie und der Subkutis ein kaliberstarker Perforator für eine etwaige Hautinsel auf.
Entsprechend dem Foramen nutricium, welches zu 90 $\%$ im mittleren Drittel der Fibula liegt und zur Sicherung sowohl der medullären als auch periostalen Blutversorgung dient, wird die Entnahmeregion im mittleren Drittel geplant [31].
Operatives Beispiel der Hebung einer vaskularisierten Fibula zur Rekonstruktion einer Pseudarthrose am Radius

Die Entscheidung zur Rekonstruktion mit einer vaskularisierten Fibula wurde in diesem Fall nach mehrfachen frustranen Versuchen nach vorangegangener Anlagerung von Beckenkammspongiosa und nachfolgendem Plattenbruch gefällt ( $\triangleright$ Abb.3-23). 


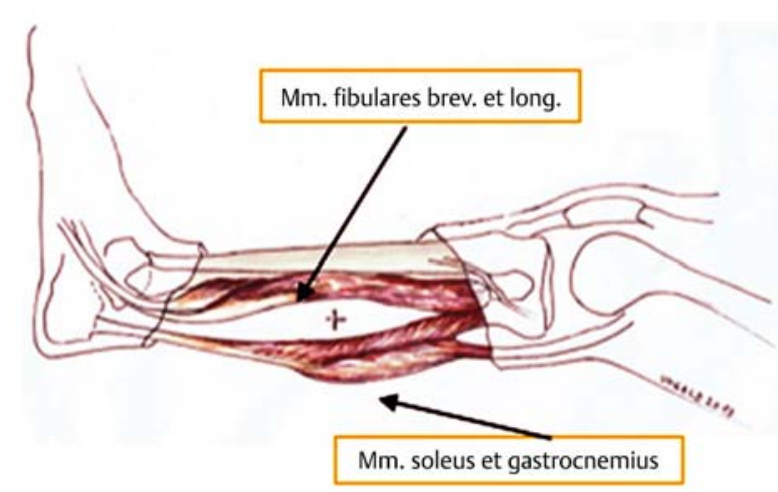

Abb.6 Schematische Darstellung des Zugangswegs von lateral.
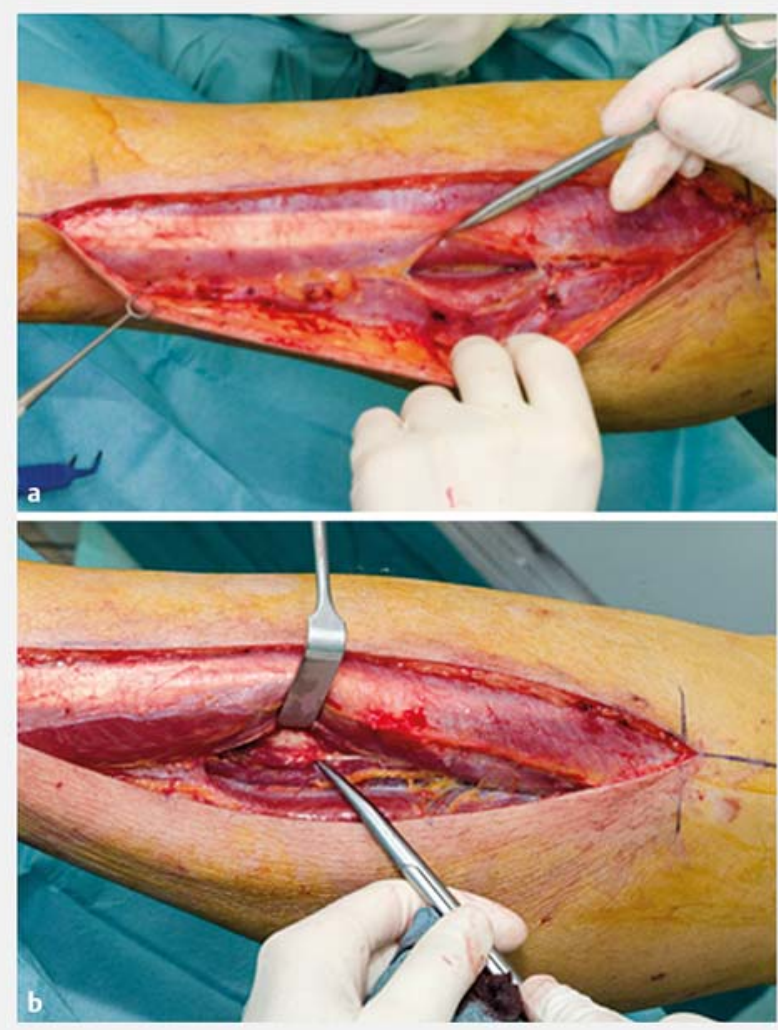

- Abb. 7 a Eingehen in der Schicht zwischen M. fibularis und M. soleus. Sichtbare unterschiedliche Zeichnung der Muskulatur und in der Tiefe tastbare und sichtbare laterale Fibulakante. b Freigelegte laterale Fibulakante.

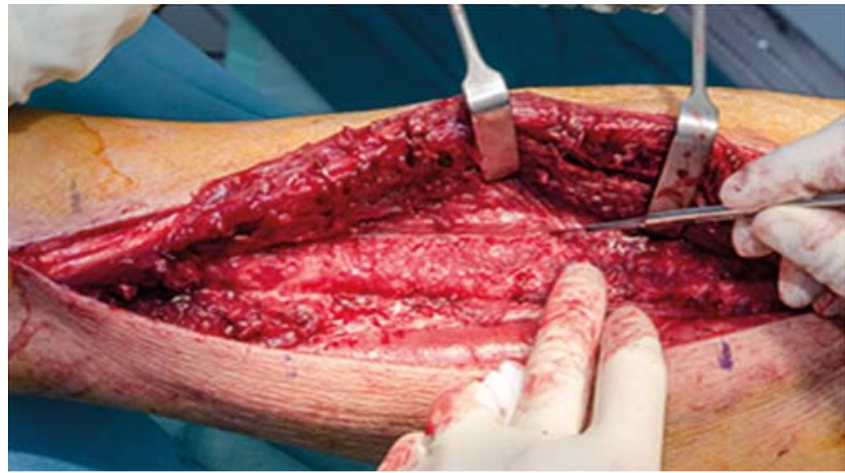

- Abb. 8 Freigelegte laterale Fibulakante mit verbliebenem schmalen Muskelrasen. Inzision der Faszie (Septum intermusculare anterius) zum M. extensor digitorum longus und M. extensor hallucis longus.

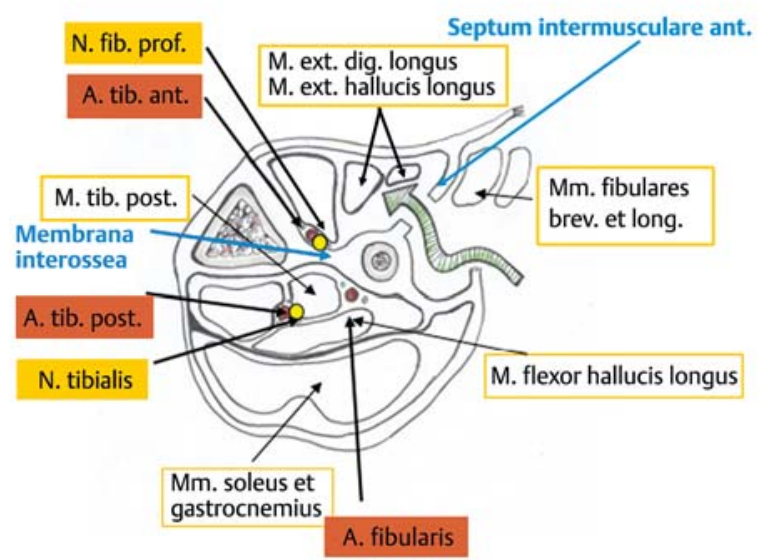

- Abb.9 Schematische Darstellung des Zugangswegs (Pfeil) durch die Septen bzw. Kompartimente des Unterschenkels im Querschnitt.

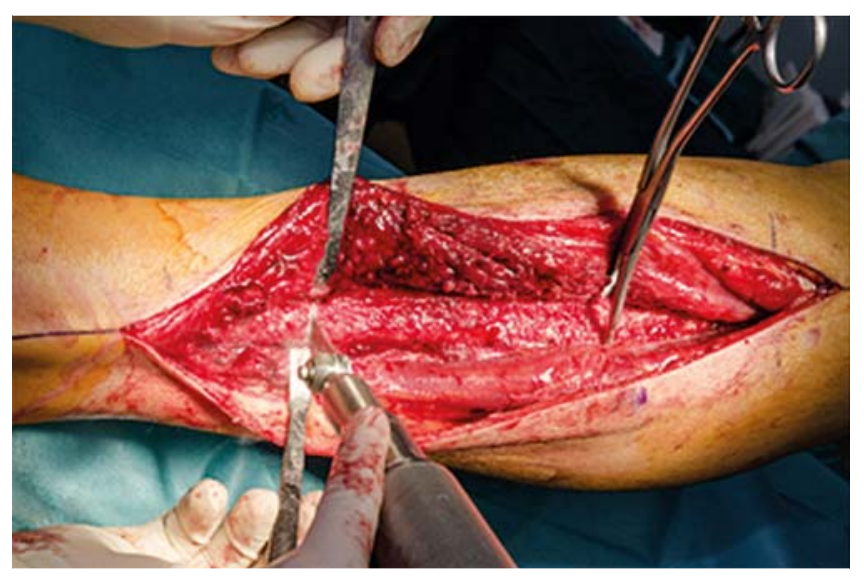

- Abb.10 Nach Abmessen des Defekts Osteotomie der Fibula mit der oszillierenden Säge und Fassen mit scharfen Knochenklemmen. 


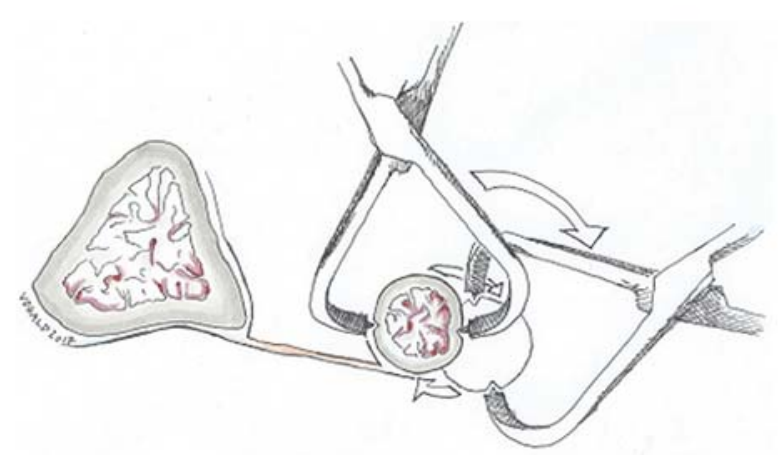

Abb. 11 Darstellung der Rotation der Fibula nach lateral nach Absetzen des Transplantats mit der oszillierenden Säge.

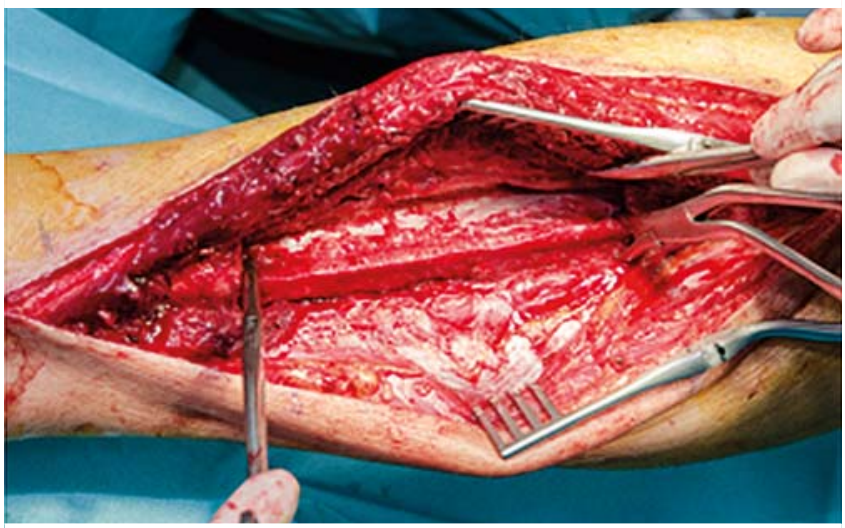

- Abb.13 Nach weiterem Rotieren der Fibula zeigt sich nun die Membrana interossea, die 2-3 mm vor der Fibulakante inzidiert wird. Oberhalb in der Muskulatur sichtbare A. tibialis anterior und Begleitvenen sowie der N. fibularis profundus.

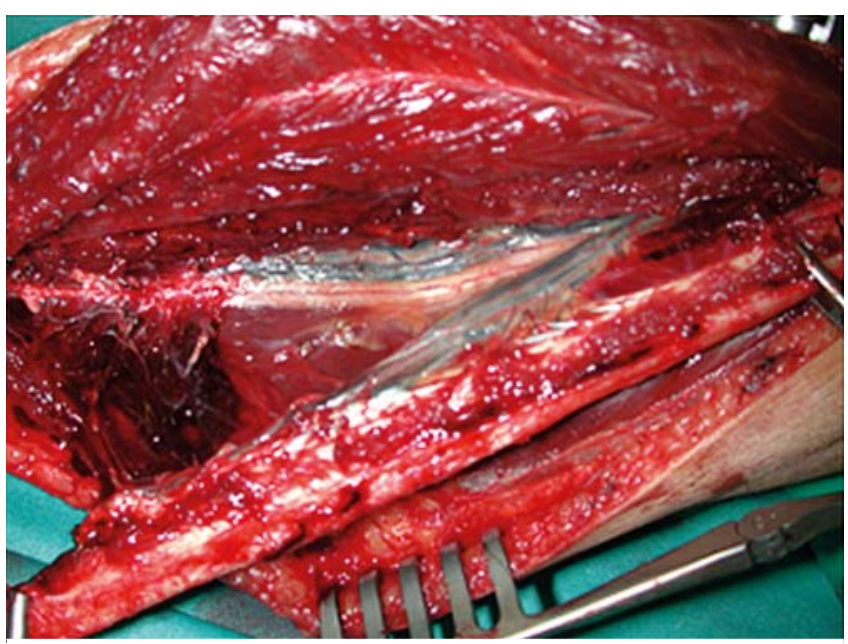

- Abb.14 Zu Demonstration der durchaus sehr variablen Anatomie möchten wir hier in einem vergleichbaren Fall einen engen $\mathrm{Ge}$ fäßverlauf der A. tibialis posterior mit dem N. tibialis unmittelbar angrenzend an die A. fibularis zeigen. Die intraoperative Präparation sollte dementsprechend sauber erfolgen und vor Absetzen des Gefäßstiels die Strukturen sicher identifiziert und mit Loops angeschlungen werden.

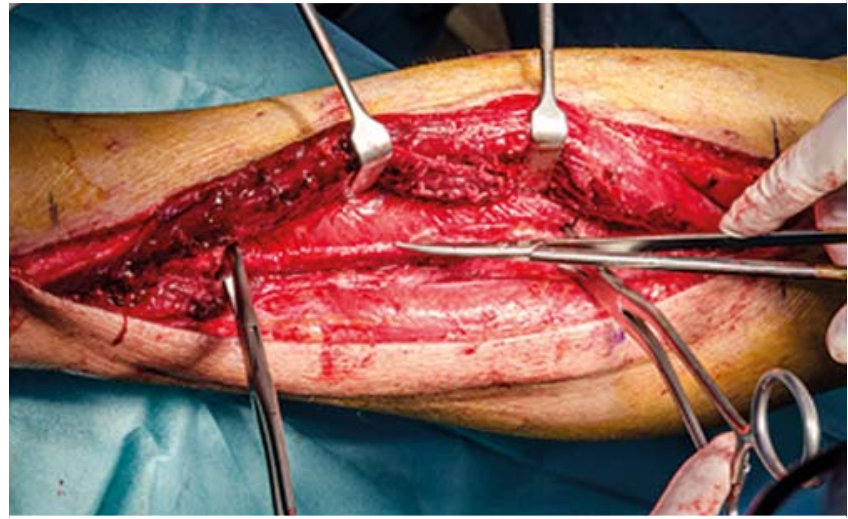

Abb.12 Rotieren der Fibula nach außen, wobei sich die dahinterliegende Muskulatur des M. extensor digitorum longus und M. extensor hallucis longus anspannt. Freipräparation der Fibula von der Muskulatur unter Belassen eines schmalen Muskelrasens.

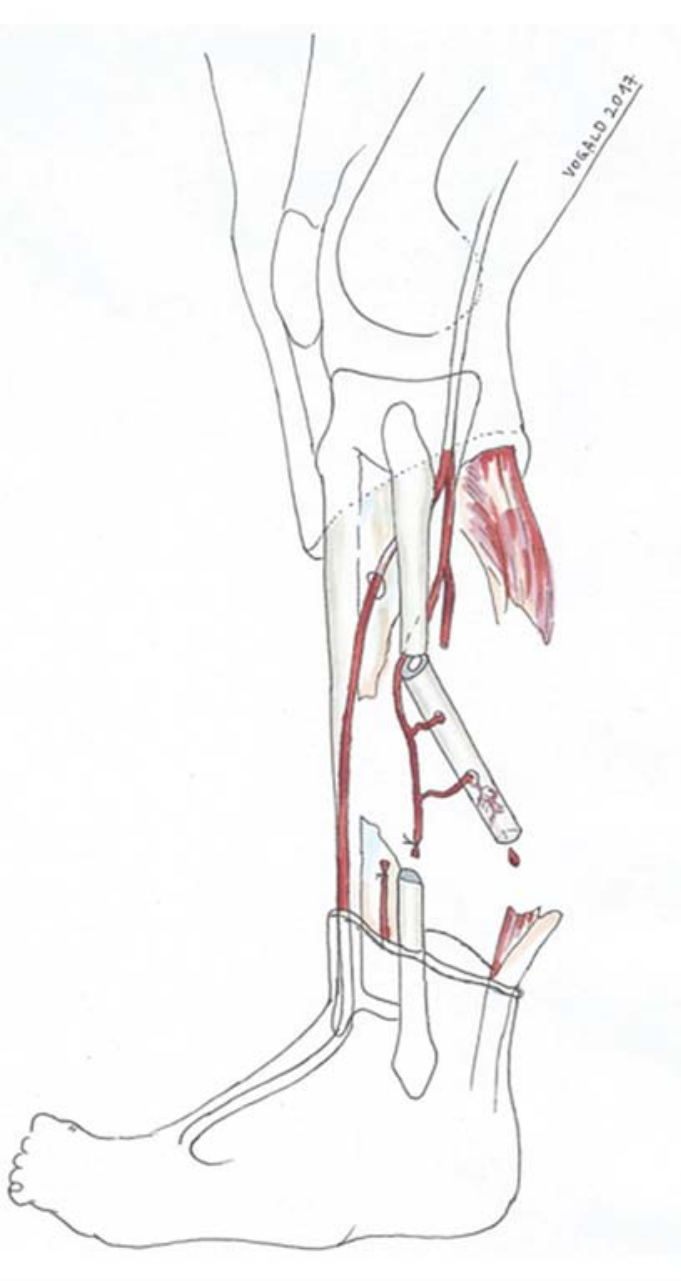

- Abb.15 Darstellung der bereits distal vom Gefäßstiel der A. fibularis gelösten Fibulatransplantats im mittleren Drittel des Unterschenkels. 


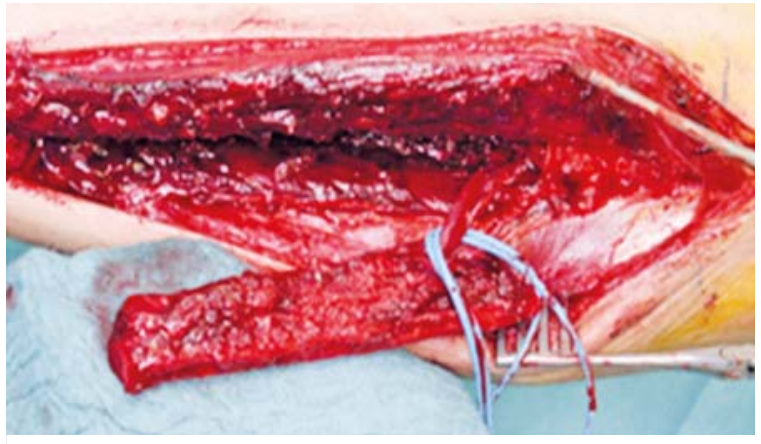

- Abb.16 Herausgelöstes Fibulatransplantat proximal am Gefäßstiel der A. fibularis hängend. Unterhalb des Gefäßstiels sichtbare Muskulatur des M. flexor hallucis sowie der darüberliegende M. tibialis posterior.

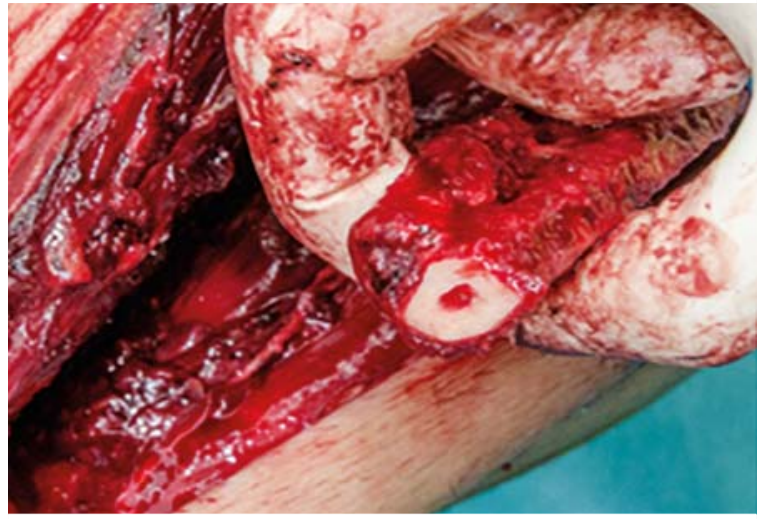

- Abb.17 Sichtbare gute endostale Durchblutung des Transplantats.
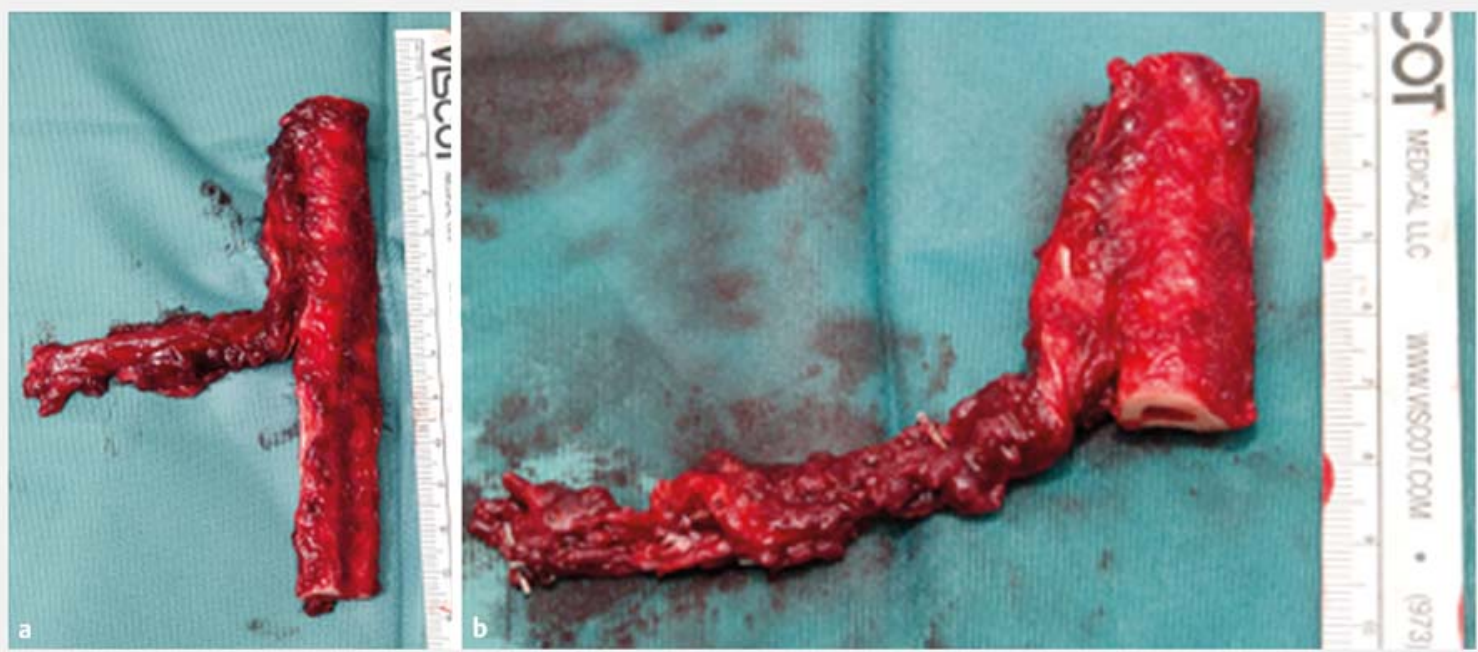

Abb. 18 Vorbereitung und Zuschneiden des Transplantats und der notwendigen Stiellänge. a Ursprüngliches Präparat. b Nach Zuschneiden der notwendigen Stiellänge.

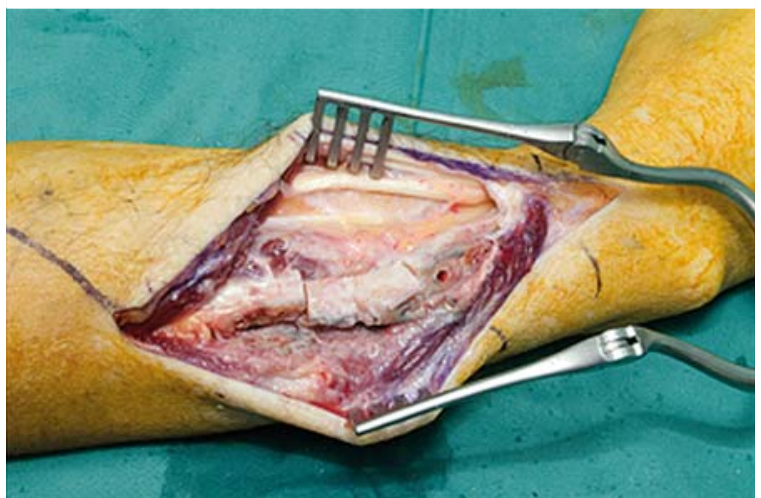

Abb.19 Resektion der Pseudarthrose am Unterarm.

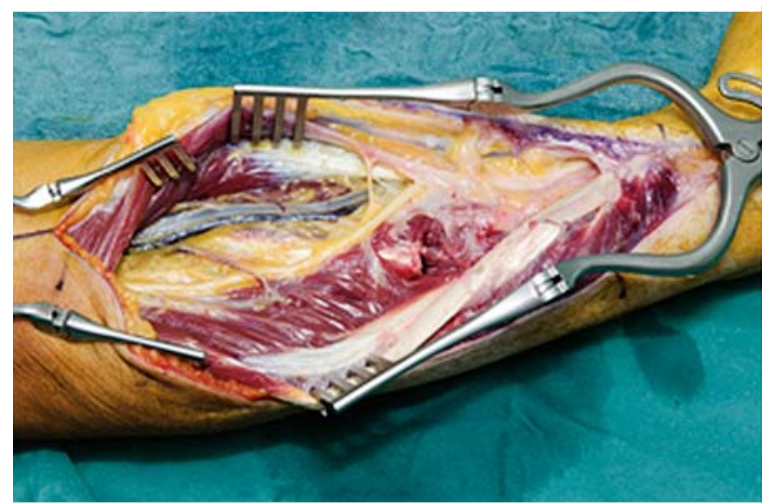

- Abb. 20 Darstellung der A. radialis für eine mikrovaskuläre End-zu-Seit-Anastomose am Unterarm. 


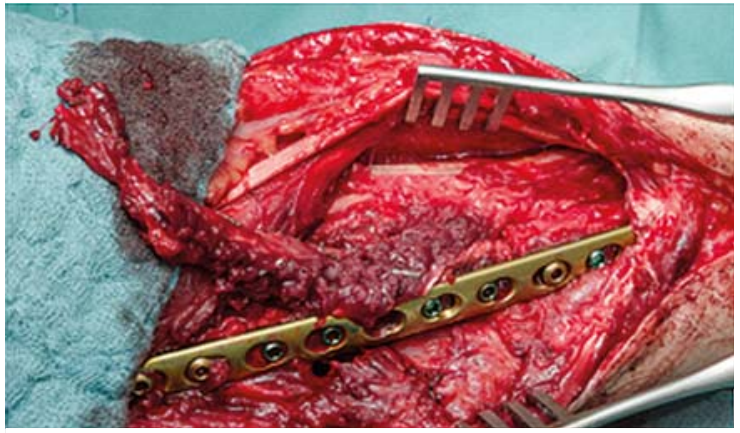

Abb. 21 Einpassen und Fixieren mit stabiler Plattenosteosynthese, anschließend Öffnen der Blutsperre und Durchführen der Gefäßanastomose.

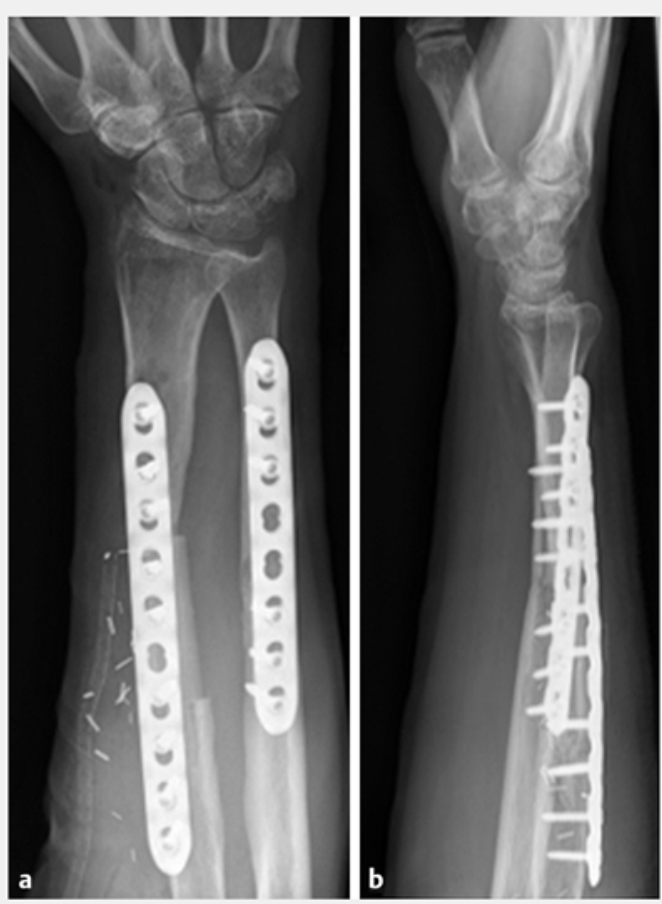

- Abb. 22 Postoperative Röntgenbilder nach Einpassen der Fibula. a Dorsopalmare Aufnahme. b Seitliche Aufnahme.

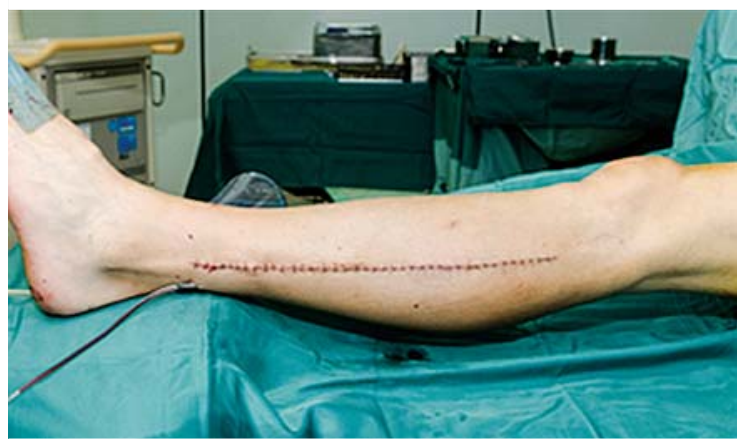

- Abb. 23 Hautverschluss unter Einlage einer RedonDrainage am Unterschenkel.

\section{ZUSATZINFO}

\section{Fehler/Gefahren}

- Fertigstellung der Osteosynthese vor den mikrovaskulären Gefäßanastomosen, da sonst das Risiko besteht, keine spannungsfreien Anastomosenverhältnisse zu erhalten [32].

- Vor dem Absetzen des Transplantats sollte eine mindestens 30-minütige Perfusion erfolgen. Andersfalls besteht ein vermehrtes Risiko von Muskelnekrosen und/oder eines avaskulären Transplantats [32].

- Unzureichende Planung, welche die OP-Zeiten verlängert, wie z. B. durch zusätzliche Venenentnahme oder kurzfristige Wechsel des Implantats oder eine zusätzliche Beckenkamminterposition bei unzureichender Länge der Fibula. Durch lange OP-Zeiten kann eine Gefährdung der Durchblutung der Extremität resultieren. Gleiches gilt für die akzidentelle Verletzung der Gefäße mit zusätzlicher Gefäßnaht.

- Die begleitende Hautinsel kann abscheren (Torsion des Gefäßstiels) oder durch zu stark komprimierende Verbände eine Durchblutungsstörung aufweisen, ohne dass eine Insuffizienz der Durchblutung des Knochentransplantats besteht.

\section{Fazit}

Im Vergleich zu den Ergebnissen des langstreckig avaskulären Knochenersatz an den Extremitäten kann das Verfahren der freien vaskularisierten Knochentransplantation insbesondere an den langen Röhrenknochen mittels freiem vaskularisiertem Fibulatransplantat eine sichere und schnellere Einheilung nach Trauma, chronischem Infekt, Tumoren und kongenitalen Pseudarthrosen gewährleisten [33]. Die Voraussetzung zur weitgehend komplikationslosen Anwendung ist die Versorgung in einem erfahrenen, mikrochirurgisch versierten Zentrum mit ausreichender präoperativer Planung, guter Patientenvorbereitung und entsprechender langfristiger Nachbetreuung. 


\section{KERNAUSSAGEN}

- Die freie, vaskularisierte Fibulatransplantation (fvF) eignet sich zur langstreckigen knöchernen Rekonstruktion von kongenitalen, onkologischen oder traumatischen Defekten sowie insbesondere auch zum Erhalt der Extremität nach Osteomyelitis.

- Die freie Fibula als vaskularisiertes Transplantat ist ein vitaler, kortikaler Knochen mit endostaler und periostaler Blutzirkulation, der auf biologische Belastungen wie ein normaler Knochen reagiert.

- Die freie vaskularisierte Fibula heilt dementsprechend nach den Gesetzen der Frakturheilung und reagiert als Transplantat mit einer Dickenzunahme sowie einer normalen Infektresistenz.

- Der Durchmesser der Fibula von ca. 1,5 cm und die Entnahmelänge von bis zu $25-30 \mathrm{~cm}$ zeigen sich besonders günstig für die direkte Rekonstruktion der Ulna und des Radius. Sie passt außerdem ideal in den medullären Raum des Humerus. Der zusätzlich hohe Anteil an kortikalem Knochen gewährleistet eine gute Stabilität bei Rotationsbelastung.

- Aufgrund der Blutversorgung sowohl endostal als auch periostal sind knöcherne Anpassungen durch Osteotomie in Längsrichtung als auch ClosedWedge-Osteotomien zur Rekonstruktion von kom- plexen anatomischen Verhältnissen unter Erhalt der Durchblutung möglich.

- Ein zuvor im Empfängergebiet durchzuführendes Débridement sollte entsprechend der Grundlage aller Infektsanierungen eine radikale Resektion avitalen Knochen- und Weichteilgewebes beinhalten.

- Kontraindikationen einer freien vaskularisierten Fibulatransplantation sind:

- ausgeprägte Gefäßveränderungen im Spenderund Empfängergebiet, vor allem eine 50 \%ige Stenose eines der versorgenden US-Gefäße [25],

- systemische Gefäßerkrankungen,

- fehlende Operabilität des Patienten.

- Es besteht die Möglichkeit der Entnahme einer Muskel-/Hautmanschette als freies, vaskularisiertes, osteokutanes Fibulatransplantat. Die maximale Größe der Hautinsel kann ca. 20 cm Länge und Breite von $10 \mathrm{~cm}$ betragen.

- Fertigstellung der Osteosynthese vor den geplanten Gefäßanastomosen, aufgrund des Risikos, möglicherweise keine spannungsfreien Anastomosenverhältnisse zu erhalten.

\section{Schlüsselwörter}

freie vaskularisierte Fibula, freies gefäßgestieltes Transplantat, gefäßgestieltes autogenes Transplantat, freies kortikales Transplantat, langstreckige Pseudarthrose, Extremitätenerhalt bei Osteomyelitis, Trauma oder Tumorresektion, Rekonstruktion langstreckiger Knochendefekte, Knochendefektüberbrückung

\section{Interessenkonflikt}

Die Autoren geben an, dass kein Interessenkonflikt besteht.

\section{Autorinnen/Autoren}

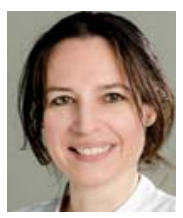

\section{Dr. Ariane Asmus}

Dr. med. 1990-1996 Studium Humanmedizin an der Freien Universität und Charité Berlin. 1997 Approbation. 2003 Facharztanerkennung Chirurgie. 2009 Schwerpunkbezeichnung Unfallchirurgie. 2013 Zusatzbezeichnung Handchirurgie. 2013 Promotion. 2014-2015 Oberärztin der Universitätsmedizin Greifswald, Abteilung für Hand- und funktionelle Mikrochirurgie. Seit 2015 Oberärztin im Unfallkrankenhaus Berlin, Hand-, Replantations- und Mikrochirurgie. 2016 EBHS Diploma - European Board of Hand Surgery Examination.

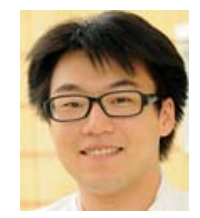

\section{Simon Kim}

Dr. med. 2003-2011 Medizinstudium Charité Berlin, University of Aberdeen (Schottland), Seoul National University College of Medicine (Südkorea). 2016 Promotion. 2012-2013 Assistenzarzt in der Abteilung für Obere Extremität des Immanuel Krankenhauses Berlin. Seit 2013 Assistenzarzt an der Klinik für Unfallchirurgie der Universitätsmedizin Greifswald.

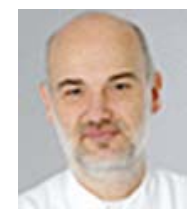

\section{Jens-Ingmar Höpfner}

Dr. med. 1985-1992 Studium an der FU Berlin, 1992 Approbation, 1998 FA Orthopädie, Oberarzt und stellvertr. Chefarzt im Unfallkrankenhaus Berlin Abt. Hand-, Replantations- und Mikrochirurgie seit 2001, Promotion 2003, Zusatzbezeichnung Handchirurgie 2006 


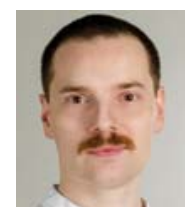

\section{Arnulf Vogel}

2000-2006 Studium Heinrich-HeineUniversität Düsseldorf, 2006-2008 Assistenzarzt Chirurgie Ev. Krankenhaus Mettmann (Dres. Kurpreugsch/Nassar/ Knaust), Unfallkrankenhaus Berlin: 2008-2010 Behandlungszentrum Rückenmarkverletzte (Dr. Niedeggen), 2010-2015 Klinik Orthopädie und Unfallchirurgie (Prof. Ekkernkamp), seit 2016 Abt. Hand-, Replantations- und Mikrochirurgie (Prof. Eisenschenk), 2018 FA Orthopädie/Unfallchirurgie, 2018 Hand- und funktionelle Mikrochirurgie Universitätsmedizin Greifswald.

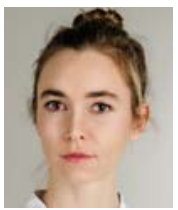

\section{Karla Vogel}

Dr. med., 2005-2012 Studium Humanmedizin Universität zu Köln, 2013 Promotion, 2013-2015 Assistenzärztin Klinik für Unfallchirurgie, Orthopädie und Handchirurgie Vivantes Klinikum am Urban Berlin (Prof. Hakimi), seit 2016 Assistenzärztin Abt. für Hand-, Replantations- und Mikrochirurgie Unfallkrankenhaus Berlin (Prof. Eisenschenk), 2018 Klinik und Poliklinik für Unfall-, Wiederherstellungschirurgie und Rehabilitative Medizin Universitätsmedizin Greifswald (Prof. Ekkernkamp).

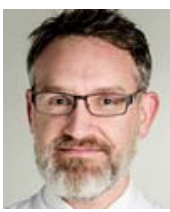

\section{Frank Eichenaue}

1993-1994 Ausbildung zum medizinisch-technischen Radiologie Assistenten. 1996-2003 Studium Humanmedizin in Berlin. 2011 Facharzt für Unfallchirurgie und Orthopädie. 2014 Zusatzbezeichnung Handchirurgie. Seit 2014 Abteilung für Hand-, Replantations- und Mikrochirurgie des Unfallkrankenhauses Berlin, seit 2015 Oberarzt.

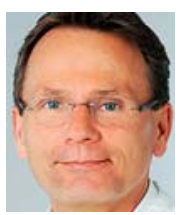

\section{Andreas Eisenschenk}

Prof. Dr. med. 1977-1984 Medizinstudium in Berlin. 1984 Approbation. 1985 Promotion. 1991 Facharzt für Orthopädie. 1992 Zusatzbezeichnung Sportmedizin. 1996 Weiterbildung zur Speziellen Orthopädischen Chirurgie. 1996 Zusatzbezeichnung Handchirurgie. 1997 Habilitation. 2007 Facharzt für Orthopädie und Unfallchirurgie. 2008 Zusatzbezeichnung Röntgendiagnostik. Seit 1997 Leiter der Abteilung für Hand-, Replantations- und Mikrochirurgie, Unfallkrankenhaus Berlin.

\section{Korrespondenzadresse}

Dr. med. Ariane Asmus

Abt. für Hand-, Replantations- und Mikrochirurgie Unfallkrankenhaus Berlin

Warener Str. 7

12683 Berlin

E-Mail: ariane.asmus@ukb.de
Wissenschaftlich verantwortlich gemäß Zertifizierungsbestimmungen

Wissenschaftlich verantwortlich gemäß Zertifizierungsbestimmungen für diesen Beitrag ist Dr. Ariane Asmus, Berlin.

Literatur

[1] Taylor GI, Miller GD, Ham F]. The Free Vascularized Bone Graft - A Clinical Extension of Microvascular Techniques. Plast Reconstr Surg 1975; 55: 533 - 544

[2] Allieu Y, Gomis R, Yoshimura M et al. Congenital pseudarthrosis of the forearm - Two cases treated by free vascularized fibular graft. J Hand Surg Am 1981; 6: 475-481

[3] Hurst LC, Mirza MA, Spellman W. Vascularized fibular graft for infected loss of the ulna: Case report. J Hand Surg Am 1982; 7: 498- 501

[4] Ueba Y, Fujikawa S. Nine years follow-up of a vascularized fibular graft in neurofibromatosis. A case report an literature review (in Japanese). Orthop Traum Surg 1983; 26 : $595-600$

[5] Muramatsu K, Hashimoto T, Tominaga Y et al. Vascularized Bone Graft for Oncological Reconstruction of the Extremities: Review of the Biological Advantages. Anticancer Res 2014; 34: $2701-2708$

[6] Pho RWH, Patterson MH, Kour AK et al. Free Vascularized Epiphyseal Transplantation in Upper Extremity Reconstruction. J Hand Surg 1988; 13-B: 440-447

[7] Innocenti M, Delcroix L, Romano GF. Epiphyseal Transplant: Harvesting Technique of the Proximal Fibula Based on the Anterior Tibial Artery. Microsurgery 2005; 25: 284-292

[8] Hankiss J, Holdenried M. Möglichkeiten und Ergebnisse der (freien) vaskularisierten Knochentransplantation im Oberund Unterarmbereich. Obere Extremität 2011; 6: 177-188

[9] Bayne CO, Bishop AT, Shin AY. Technique of Harvest of the Free Vascularized Fibula. Tech Hand Up Extrem Surg 2014; 18: $181-188$

[10] De Boer HH, Wood MB. Bone Changes in the Vascularised Fibular Graft. J Bone Joint Surg 1989; 71-B: $374-378$

[11] Weiland AJ, Phillips TW, Randolph MA. Bone Grafts: A radiologic, Histologic, and Biomechanical Model Comparing Autografts, Allografts, and Free Vascularized Bone Grafts. Plast Reconstr Surg 1984; 74: 368-379

[12] Eisenschenk A, Lehnert M, Weber U. Die freie, gefäßgestielte Fibulatransplantation zur Überbrückung von Knochendefekten. Operat Orthop Traumatol 1994; 6: 107-118

[13] Eisenschenk A. Tierexperimentelle und klinische Untersuchungen der Knochenheilung vaskularisierter Knochentransplantate in Abhängigkeit verschiedener EinflußgröBen. HabilitationsschriftFU Berlin; 1996

[14] Eisenschenk A, Lautenbach M, Rohlmann A. Freie, vaskularisierte Knochentransplantation im Bereich der Extremitäten. Orthopäde 1998; 27: $491-500$

[15] Arata MA, Wood MB, Cooney WPIII. Revascularized segmental diaphyseal bone transfers in canine. An analysis of viability. J Reconstr Microsurgery 1984; 1: 11-19

[16] Cutting CB, Mc Carthy JG. Comparison of residual osseus mass between vascularized and nonvascularized onlay bone transfers. Plast Reconstr Surg 1983; 72: 672-675 
[17] Taylor GI. The Current Status of Free Vascularized Bone Grafts. Clin Plast Surg 1983; 10: 185- 209

[18] Wood MB. Upper Extremity Reconstruction by Vascularized Bone Transfers: Results and Complications. J Hand Surg 1987; 12-A: $422-501$

[19] Schuind FA, Burny F, Lejeune FJ. Microsurgical Free Fibular Bone Transfer: A Technique for Reconstruction of Large Skeletal Defects Following Resection of High-Grade Malignant Tumors. World J Surg 1988; 12: 310-317

[20] Malizos KN, Zalavras CG, Soucacos PN et al. Free Vascularized Fibular Grafts for Reconstruction of Skeletal Defects. J Am Acad Orthop Surg 2004; 12: 360 - 369

[21] Wolff KD, Hölzle F. Raising of Microvascular Flaps. A Systematic Approach2nd ed. Berlin: Springer; 2005

[22] Shammas RL, Avashia Y], Farjat AE et al. Vascularized FibulaBased Physis Transfer: A Follow-Up Study of Longitudinal Bone Growth and Complications. Plast Reconstr Surg Global Open 2017; 5: e1352

[23] Ekkernkamp A, Muhr G, Josten C. Die infizierte Pseudarthrose. Unfallchirurg 1996; 99: 914-924

[24] Lutz B, Wei FC, Ng SH et al. Routine Donor Leg Angiography before Vascularized Free Fibula Transplantation Is Not Necessary: A Prospective Study in 120 Clinical Cases. Plast Reconstr Surg 1998; 103: $121-127$

[25] Oxford L, Ducic Y. Use of Fibula-Free Tissue Transfer With Preoperative 2-Vessel Runoff to the Lower Extremity. Arch Facial Plast Surg 2005; 7: $261-264$

[26] Hierner R, Täger G, Nast-Kolb D. Die vaskularisierte Knochentransplantation. Unfallchirurg 2009; 112: 405-418
[27] Godina M. Early Microsurgical Reconstruction of Complex Trauma. Plast Reconstr Surg 1986; 78: 285-292

[28] Höpfner Jl. Klinische und radiologische Langzeitergebnisse nach freier, vaskularisierter Knochentransplantation zur Überbrückung großer Defekte der langen Extremitätenknochen. Inaugural-DissertationFU Berlin; 2003

[29] Han CS, Wood MB, Bishop AT et al. Vascularized Bone Transfer. J Bone Joint Surg 1992; 74-A: 1441 - 1449

[30] Hirayama T, Suematsu N, Inoue K et al. Free Vascularised Bone Grafts in Reconstruction of the Upper Extremity. J Hand Surg 1985; 10-B: 169- 175

[31] Hierner R, Stock W, Wood MB et al. Der vaskularisierte Fibulatransfer. Unfallchirurg 1992; 95: 152 - 159

[32] Eisenschenk A, Lehnert M, Weber U. Die freie, gefäßgestielte Fibulatransplantation zur Überbrückung von Knochendefekten. Operat Orthop Traumatol 1994; 6: 107-118

[33] Enneking WF, Eady JL, Burchardt H. Autogenous Cortical Bone Grafts in the Reconstruction of Segmental Skeletal Defects. J Bone Joint Surg 1980; 62-A: 1039-1057

Bibliografie

DOI https://doi.org/10.1055/s-0043-124764

Handchirurgie Scan 2018; 07: 215-229

(c) Georg Thieme Verlag KG Stuttgart · New York ISSN 2194-8976 


\section{Punkte sammeln auf CME.thieme.de}

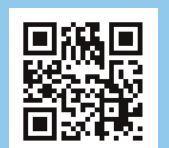

Diese Fortbildungseinheit ist 12 Monate online für die Teilnahme verfügbar.

Sollten Sie Fragen zur Online-Teilnahme haben, finden Sie unter http://cme.thieme.de/hilfe eine ausführliche Anleitung. Wir wünschen viel Erfolg beim Beantworten der Fragen!

Unter https://eref.thieme.de/u2d003 oder über den QR-Code kommen Sie direkt zum Artikel zur Eingabe der Antworten.

VNR 2760512018154651681

\section{Frage 1}

Welches ist keine Voraussetzung für eine präoperative Angiografie der Beine?

A Unauffällige Fußpulse bei Palpation oder Doppler-Untersuchung.

B Trauma der oder des Unterschenkels in der Anamnese.

C Zeichen der Mikro- oder Makroangiopathie in der Anamnese.

D Zustand nach Bypassoperation.

E Narben im Spendergebiet.

\section{Frage 2}

Welche präoperative Untersuchung gehört nicht zum Standard vor einer freien, vaskularisierten Fibulatransplantation?

A Röntgenbilder des Entnahme- und Spendergebiets.

B Angiografie des Empfängergebiets.

C CT des Spendergebiets.

D Palpation oder Doppler-Untersuchung der Fußpulse.

E Nachweis der Infektfreiheit im Empfängergebiet.

\section{Frage 3}

Welche Aussage ist richtig?

A Die Blutversorgung der Fibula erfolgt ausschließlich endostal.

B Die Blutversorgung der Fibula erfolgt endostal und periostal.

C Die Blutversorgung der Fibula erfolgt ausschließlich periostal.

D Die Blutversorgung der Fibula erfolgt über myokutale Perforansgefäße.

E Der Blutfluss verläuft zentripetal von außen (Kortikalis) nach innen (Spongiosa).

\section{Frage 4}

Wie viele cm sollten bei der Entnahme der Fibula proximal belassen werden?

$\begin{array}{lr}\text { A } & 2 \mathrm{~cm} \\ \text { B } & 4 \mathrm{~cm} \\ \text { C } & 6 \mathrm{~cm} \\ \text { D } & 8 \mathrm{~cm} \\ \text { E } & 10 \mathrm{~cm}\end{array}$

\section{Frage 5}

In wieviel Prozent der Fälle liegt das Foramen nutricium im mittleren Drittel der Fibula?

A in weniger als $20 \%$ der Fälle

$B$ in $30 \%$ der Fälle

C in der Hälfte der Fälle

D in $70 \%$ der Fälle

$\mathrm{E}$ in $90 \%$ der Fälle

\section{Frage 6}

Wie viele cm sollten bei der Entnahme der Fibula distal belassen werden?
A $\quad 2 \mathrm{~cm}$
B $\quad 4 \mathrm{~cm}$
C $6 \mathrm{~cm}$
D $8 \mathrm{~cm}$
E $10 \mathrm{~cm}$

\section{Frage 7}

Zwischen welchen Muskeln wird beim lateralen Zugang von Gilbert eingegangen?

A M. peronaeus longus und $M$. soleus

B Mm. fibulares brevis et longus und $\mathrm{Mm}$. soleus et gastrocnemius

C M. gastrocnemius und $M$. peronaeus brevis

D $M$. soleus und $M$. gastrognemius

E M. fibularis brevis und M. peronaeus brevis

\section{Frage 8}

Welche Aussage trifft nicht zu? Kontraindikationen zur Transplantation einer vaskularisierten Fibula sind...

A begleitende Weichteildefekte.

B ausgeprägte Gefäßveränderungen im Spendergebiet.

C systemische Gefäßerkrankungen.

D ausgeprägte Gefäßveränderungen im Empfängergebiet.

E fehlende Operabilität des Patienten bei schweren Allgemeinerkrankungen. 


\section{Punkte sammeln auf CME.thieme.de}

Fortsetzung ...

\section{Frage 9}

Welche Antwort trifft nicht zu? Welcher Punkt sollte im Rahmen der Patientenaufklärung Erwähnung finden?

A Antikoagulation mit niedermolekularem Heparin

B Schwellung und Nachblutung im Spendergebiet

C unschöne Hebedefekte und vermehrte Narbenbildung nach Spalt- bzw. Vollhautentnahme

D Kraftminderung und Beugeschwäche der Großzehe nach Entnahme einer zusätzlichen Muskelmanschette

E Immobilisierung der Spenderextremität

\section{Frage 10}

Welche Aussage bez. der Entnahmeregion trifft zu?

A Die Entnahmeregion wird im distalen Drittel geplant.

B Die Entnahmeregion wird im proximalen Drittel geplant.

C Die Entnahmeregion wird im mittleren Drittel geplant.

D Die Entnahmeregion kann überall gewählt werden.

E Bei Kindern sollte die Entnahmeregion standardmäßig weit distal gewählt werden. 